\title{
ON THE TURBULENT VISCOSITY PRESCRIPTION IN ACCRETION DISCS
}

\author{
PATRICK GODON \\ NASA/Jet Propulsion Laboratory, 4800 Oak Grove Dr., \\ MS 238-332, Pasadena, CA 91109, USA
}

\begin{abstract}
The validity of the standard $\alpha$ viscosity prescription in turbulent accretion discs is analyzed. It is found that the $\alpha$ viscosity prescription is no longer valid when the disc is not Keplerian. The analysis is based on simple scaling arguments for fully developed turbulences in accretion discs. An analytical treatment is carried out for geometrically thin, non-Keplerian discs in vertical hydrostatic equilibrium, and is compared with the standard values of the viscosity in the boundary layer region and with the viscosity prescription derived from a general causal equation for the evolution of the stress tensor.
\end{abstract}

\section{Introduction}

Instability analyses and numerical simulations have associated the origin of instabilities in accretion discs to the $\alpha$ viscosity prescription. Three recent works (Narayan, Loeb \& Kumar 1994; Papaloizou \& Szuszkiewicz 1994; Kato \& Inagaki 1994) have dealt with the problem of the viscosity in rotating flows in detail. Their results show that the viscosity law for a non-Keplerian disc cannot be represented by the standard $\alpha$ viscosity prescription. In this work, we develop a turbulent viscosity prescription for non-Keplerian discs, using simple scaling arguments. We find very good agreement with the results of the three recent works mentioned above.

\section{The standard $\alpha$ viscosity prescription}

In the theory of fully developed turbulences (Landau \& Lifshitz 1987), the viscosity is given by $\nu=a \Delta u \lambda$, where $a$ is a constant of order unity, $\Delta u$ is the change of the flow mean velocity $u$ over a distance $\lambda$ - the scale length of the largest eddies, responsible for the transport of angular momentum. 
For a geometrically thin, Keplerian disc in vertical hydrostatic equilibrium one obtains: $\lambda=H<<r, u=v_{\mathrm{K}}, \Delta u=v_{\mathrm{K}} H / 2 r, H / r=v_{\mathrm{s}} / v_{\mathrm{K}}$ and

$$
\nu=a v_{\mathrm{s}} H / 2 \text {. }
$$

\section{Turbulent viscosity prescription for non-Keplerian discs}

In this case one expects $\lambda=\bar{H}=P /|\nabla P|$ (Papaloizou \& Stanley 1986) and $\Delta u(\lambda)=r\left|\Omega^{\prime}\right| \lambda$. Assuming $\Omega^{\prime} \neq 0$ leads to

$$
\nu=a v_{\mathrm{s}} H \frac{\left|\Omega^{\prime}\right|}{\left|\Omega_{\mathrm{K}}^{\prime}\right|}(\bar{H} / H)^{2}=a v_{\mathrm{s}} H \mathcal{M}_{\mathrm{t}}^{2} \frac{\left|\Omega^{\prime}\right|}{\left|\Omega_{\mathrm{K}}^{\prime}\right|},
$$

where constant terms of order unity have been included in $a$ and $\mathcal{M}_{\mathrm{t}}$ is the turbulent Mach number. For convenience we have written the viscosity in the form $\nu=a v_{\mathrm{s}} H F$. The stress tensor is then written

$$
\tau_{r \phi}=-a P \frac{\left|\Omega^{\prime}\right|}{\left|\Omega_{\mathrm{K}}^{\prime}\right|} F
$$

where $F=\nu /\left(a v_{\mathrm{s}} H\right)$. The stress tensor vanishes whenever $\Omega^{\prime}=0$. This prescription of the viscosity is completely consistent with the treatment of Shakura \& Sunyaev (1988) for the boundary layer region, and is in good agreement with the causality formalism, in which one expects the viscosity to be reduced when the radial infall velocity increases such that $v_{\mathbf{r}} \approx \max \left(v_{\mathrm{s}}, v_{\mathrm{t}}\right)$, where $v_{\mathrm{t}}$ is the turbulent velocity [see Godon (1995) for more details]. Recent observations (Horne 1995) show supersonic turbulent velocities with $\mathcal{M}_{\mathrm{t}} \approx 10$ in accretion discs. In this case the supersonic turbulences might contribute only partially to the cascade effect, (since they also dissipate in part through shocks), and the present treatment would need improvement to take this effect into consideration.

\section{References}

Godon, P., 1995, MNRAS, 177, 157

Horne, K., 1995, poster presentation, IAU Coll. 158

Kato, S., Inagaki, S., 1994, PASJ, 46, 289

Landau, L., Lifshitz, E., 1987, Fluid Mechanics, Pergamon Press (2nd Edition), Oxford

Narayan, R., Loeb, A., Kumar, P., 1994, Ap. J., 431, 359

Papaloizou, J.C.B., Stanley, G.Q.G., 1986, MNRAS, 220, 253

Papaloizou, J.C.B., Szuszkiewicz, E., 1994 MNRAS, 268, 29

Shakura, N.I., Sunyaev, R.A., 1988, Adv. Sp. Res., 8, 135 